\section{No preservatives}

If instant coffee keeps a good part of its native virtues when it is lyophilized

(freeze-dried), why not also red blood cells? There are various ways of storing material for transfusion - wet, dry, frozen, or even perhaps in the form of cross-linked haemoglobin. Each has its advantages; but if cost is a primary criterion, R. P. Goodrich et al. (Proc. natn. Acad. Sci. U.S.A. 89, 967-971; 1992) may have the answer. For lyophilized cells, they find, can be stored at room temperature and their metabolic state is only mildly perturbed by what they have been through - no more so, at all events, than that of conventionally banked cells. It is true that the drying takes seven days of pumping, but the argument is that this will still be cheaper than maintaining refrigeration plants. Soon you may be able to carry sachets of your own dried red cells in your pocket with your car insurance and donor card.

\section{Chemistry set}

WiTH all this talk about nanotechnology (see page 761), chemists are keen to restake their natural claim to this domain. In a virtual manifesto (J. Am. chem. Soc. 114, 601-621; 1992), P. Kaszynski, A. C. Friedli and J. Michl describe their ambitions for a molecular "Tinkertoy" set, with which one could make mechanically strong designer solids as light as aerogels. Chemical substitutions would endow the products with special properties. The authors outline the synthesis of rigid molecular rods built up from individual 5-carbon, propellane molecules. They obtain these 'staffane' rods in only small yields, but find them to be stable to heat, air and common solvents, and transparent to light. Kaszynski et al. concede that synthesizing the spools needed to connect these rods will be the hard part.

\section{Wash out}

J. SMIT et al. (Geology 20, 99-103; 1992) have come up with support for the idea that the Yucatán area of Mexico was hit by a large extraterrestrial body at the Cretaceous/Tertiary boundary. The authors looked at an outcrop of rock in northeastern Mexico which dates biostratigraphically to around that time, and which was deposited under gentle conditions some $400 \mathrm{~m}$ underwater. Running through the outcrop is a distinctive unit topped by a band anomalously rich in iridium and with features consonant with there having been a disruptive event nearby: the lower part contains tektites (glass droplets) and minerals bearing the scars of impact shock; above that lie coarse sediments and plant remains. Smit et al. interpret the one as the product of the impact's immediate fall-out, the other as larger deposits from giant waves stirred up in the Gulf of Mexico.

\section{Models of the seismic cycle}

\section{Christopher H. Scholz}

COULD the Loma Prieta earthquake of 1989 have been predicted from precursory seismic activity in California? Quite possibly, suggest B. E Shaw, J. M. Carlson and J. S. Langer in the Journal of Geophysical Research (97, 479-488; 1992), on the basis of computerized simulations of a mechanical model of earthquake activity.

Models of spatially extended, dissipative systems which exhibit the property known as self-organized criticality have been widely discussed as models of earthquakes (see my News and Views article in Nature 348, 197-198; 1990). Their main claim to fame as earthquake models is that they mimic the GutenburgRichter law for the frequency of earthquakes of different sizes. There are now quite a few variations of this class of model that can join the club if this is the sole basis for membership. Shaw, Carlson and Langer delve more deeply into their particular model, attempting a much more ambitious comparison of its behaviour with natural seismicity.

Their model is a onedimensional spring-slider chain (see Fig. 1) in which the block slider motions are solved dynamically. It produces a spectrum of smallscale events that exhibit scaling (frequency reducing with size) and occasional large events which do not belong to this scaling relation. Natural earthquakes also fit into two classes, and J. F. Pacheco, L. R. Sykes and I have recently shown that large and small earthquakes have different size distributions (Nature 355, 71-73; 1992).

Shaw and colleagues compare, for their model, the temporal and spatial relationship of the small events to the large ones, showing that the small-event activity increases and becomes more

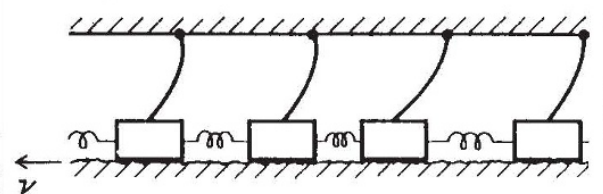

FIG. 1 A chain of sliding blocks connected by springs can behave like a geological fault (From Shaw et al.) IMAGE
UNAVAILABLE
FOR COPYRIGHT
REASONS concentrated towards the end of the large-event cycle, which might be understood as a form of precursory activity for large events. Their comparison with real earthquake catalogues gives mixed results. In general, the natural seismicity did not show any clear indication of this clustering, and in many cases evolved quite differently.

The one exception was an acceleration of activity on the Calaveras fault, connected to the San Andreas fault, in the years preceding the 1989 Loma Prieta earthquake. Long-term acceleration of activity in broad regions surrounding this and other earthquakes has been noted by L. R. Sykes and S. C. Jaumé (Nature 348, 595-599; 1990). In that study, the authors weighted the data according to earthquake moment, which favours large events, rather than event counts, as used by Shaw et al., which emphasizes smaller events. The convergence of these results, and the guidance provided by the model, suggest that a more diligent examination of seismic catalogues is now in order.

Shaw and colleagues are the first to attempt to apply this type of model to 